\title{
PRIMARY TESTICULAR LYMPHOMA: A SINGLE CENTRE EXPERIENCE
}

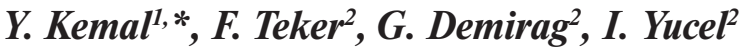 \\ ${ }^{1}$ Department of Medical Oncology, Samsun Education and Research Hospital, Samsun 55100, Turkey \\ ${ }^{2}$ Department of Medical Oncology, Faculty of Medicine, 19 Mayis University, Samsun 55139, Turkey
}

Aim: Primary testicular lymphoma (PTL) is an uncommon and aggressive form of extranodal non-Hodgkin's lymphoma (NHL). We aimed to analyse the clinicopathological characteristics and outcomes of our PTL cases. Materials and Methods: A review was made of the medical records of 339 NHL patients who were treated in the Medical Oncology Department between January 2005 and December 2013. Results: 8 PTL patients were identified from the 339 NHL patients. The average age of the patients was 67.7 \pm 7.9 years (range 53-79 years). The mean follow-up time was 24.8 months (range 7-98 months). Inguinal orchiectomy was performed as a diagnostic and initial therapy and all the patients underwent 4-6 cycles of chemoimmunotherapy consisting of cyclophosphamide, doxorubicin, vincristine and prednisone plus rituximab. 4 of 8 patients received intrathecal prophylactic chemotherapy and 6 of 8 patients continued contralateral testis irradiation. Relapse occured in only 1 patient in central nervous system after 6 months who had not received intrathecal prophylaxis. No contralateral testis relapse was observed. Conclusions: Primary testicular NHL is an uncommon entity and we evaluated 8 patients; with one relapse in central nervous system and no relapse in the contralateral testis.

Key Words: primary testicular lymphoma, contralateral scrotal irradiation, intratechal chemotherapy.

Non-Hodgkin's lymphoma (NHL) is a common disease and nearly $30 \%$ of cases occur in extranodal sites. Primary testicular lymphoma (PTL) is an uncommon and aggressive form of extranodal NHL [1, 2]. It accounts for less than $5 \%$ of testicular malignancies and $1-2 \%$ of NHL cases, although it is the most common testicular malignancy in men aged $>60$ years $[1,3]$. PTL is usually characterized by the unilateral mass or swelling of testis and synchronous bilateral involvement at presentation occurs in $6-10 \%$ of patients $[4,5]$.

Histopathologically, diffuse large B-cell lymphoma (DLBCL) is the predominant type in more than $80 \%$ of PTL cases [1]. Clinically they do not display the clear survival plateau of other high-grade lymphomas because of the occurrence of late relapses [1, $4,6]$. Relapse presents mostly in extranodal sites, especially in the central nervous system (CNS) and contralateral testis [7]. Both these sites are considered as immunoprivileged sites, owing to the blood-brain and blood-testis barrier which reduces the penetration of chemotherapy agents as lymphoma cells may escape the host T-cell antitumour response [8-10].

PTL is a rare condition and prospective randomised controlled trials have been difficult to establish, so standard treatments for PTL have not been well defined. Orchiectomy is indicated for both diagnostic and therapeutic purposes but prognosis is considered to be poor in patients treated with only orchiectomy and/or radiation $[4,11]$. Recently combined modality treatment with rituximab plus doxorubicin-based chemotherapy, prophylactic intrathecal chemotherapy,

Submitted: May 26, 2015.

${ }^{\star}$ Correspondence: E-mail: drturkmen@yahoo.com Abbreviations used: CHOP - cyclophosphamide, doxorubicin, vincristine, and prednisone; CNS - central nervous system; $\mathrm{CR}$ - complete remission; DLBCL - diffuse large B-cell lymphoma; IPI - international prognostic index; NHL - non-Hodgkin's lymphoma; OS - overall survival; PFS - progression-free survival; PTL primary testicular lymphoma; R - rituximab; RT - radiotherapy. and scrotal radiotherapy is recommended, but controversies still exist.

In this retrospective study, it was aimed to analyse the clinicopathological characteristics and outcomes of 8 PTL cases and discuss the knowledge and experience gained in this rare but important entity.

\section{PATIENTS AND METHODS}

A review was made of the medical records of $339 \mathrm{NHL}$ patients who were treated in the Medical Oncology Department between January 2005 and December 2013. Eight of the 339 patients were PTL. All PTL patients underwent orchiectomy for pathological diagnosis. The Ann Arbor classification system was used for staging and B symptoms included night sweats, recurrent fever and unexplained $10 \%$ loss of body weight within 6 months. The international prognostic index (IPI) score was also determined.

Complete remission (CR) was defined as absence of disease signs and symptoms one month after completion of all treatments. The overall survival (OS) duration was calculated from the time of diagnosis to the time of death or to the last follow-up. Progression-free survival (PFS) was measured from the time of diagnosis to the time of treatment failure, relapse, or death because of lymphoma. Relapse was defined as the appearance of a new lesion in a patient with CR.

Statistical analyses were performed with SPSS soft ware (SPSS 15.0, Chicago, IL). Descriptive analyses were used for the characteristics of the study population. Survival analysis was applied with the Kaplan Meier method.

\section{RESULTS}

Between January 2005 and December 20138 PTL patients were identified from the $339 \mathrm{NHL}$ patients. The average age of the patients was $67.7 \pm 7.9$ years (range 53-79 years). The mean follow-up time was 24.8 months (range 7-98 months). 4 patients had PTL 
in the right testicle and 4 patients had PTL in the left testicle. 6 patients were Ann Arbor stage I or II at the time of diagnosis and 2 patients were stage III. Only 1 patient had B symptoms at stage III. Inguinal orchiectomy was performed as a diagnostic and initial therapy and DLBCL was confirmed in all 8 patients after histopathological examination. After orchiectomy, all the patients underwent 4-6 cycles of chemoimmunotherapy consisting of cyclophosphamide, doxorubicin, vincristine and prednisone (CHOP) plus rituximab $(\mathrm{R})$, followed by intrathecal prophylactic chemotherapy in 4 of 8 patients and irradiation of the contralateral testis in 6 of 8 patients. CR was achieved in 7 patients after R-CHOP chemotherapy, 1 patient was still undergoing chemotherapy during the last follow-up with partial response (patient \#2) and 1 patient was continuing chemotherapy and planned contralateral testis irridation (patient \# 8). During the follow-up period, a relapse occured in only 1 patient (patient \# 4) in the CNS after 6 months, and it was determined that he had not received CNS prophylaxis. He was treated only with palliative radiotherapy. No contralateral testis relapse was observed. The patient characteristics and follow-up data are presented in Table. Figure shows the OS analysis of the PTL cases.

Table. Epidemiological features, clinicopathological characteristics, type of management, and outcome of patients with PTL

\begin{tabular}{|c|c|c|c|c|c|c|c|c|c|c|c|}
\hline 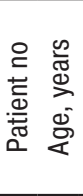 & 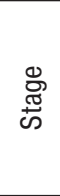 & $\overline{\underline{ }}$ & 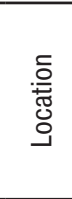 & Surgery & $\begin{array}{l}\text { Chemo- } \\
\text { therapy }\end{array}$ & เ & 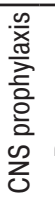 & 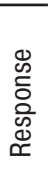 & 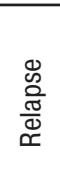 & م & \\
\hline 153 & $\mathrm{IE}$ & 1 & Left & Orchiectomy & $4 \times \mathrm{R}-\mathrm{CHOP}$ & + & $-(-1)$ & $\mathrm{CR}$ & - & 98 & \\
\hline 273 & IIIEB & 4 & Left & Orchiectomy & $4 \times \mathrm{R}-\mathrm{CHOP}$ & - & - & PR & - & 7 & \\
\hline 363 & IIIE & 4 & Right & Orchiectomy & $6 \times \mathrm{R}-\mathrm{CHOP}$ & + & + & CR & - & 9 & \\
\hline 468 & IIE & 2 & Right & Orchiectomy & $6 \times \mathrm{R}-\mathrm{CHOP}$ & + & - & CR & $+(6)$ & 12 & \\
\hline 579 & $\mathrm{IE}$ & 1 & Right & Orchiectomy & $4 \times \mathrm{R}-\mathrm{CHOP}$ & + & - & CR & - & 15 & + \\
\hline 666 & IIE & 1 & Right & Orchiectomy & $6 \times \mathrm{R}-\mathrm{CHOP}$ & + & + & CR & - & 36 & \\
\hline $7 \quad 74$ & IIE & 1 & Left & Orchiectomy & $6 \times \mathrm{R}-\mathrm{CHOP}$ & + & + & CR & - & 14 & \\
\hline 866 & $\mathrm{IE}$ & 1 & Left & Orchiectomy & $6 \times \mathrm{R}-\mathrm{CHOP}$ & - & $-(1+5)$ & $\mathrm{CR}$ & - & 8 & + \\
\hline
\end{tabular}

Note: R-CHOP - rituximab, cyclophosphamide, doxorubicin, vincristine, and prednisone; RT - radiotherapy; PR - partial remission.

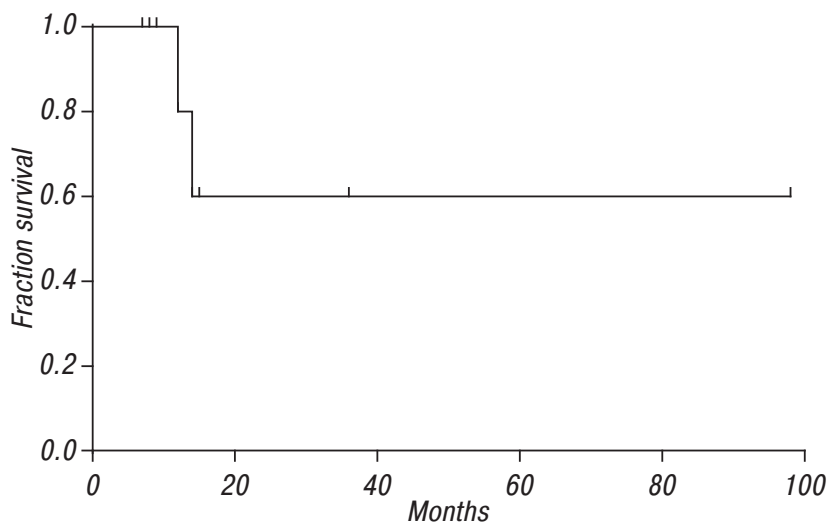

Figure. Kaplan - Meier OS curve for PTL patients

\section{Discussion}

In this study, the clinicopathological characteristics and outcomes of 8 PTL cases treated at our department have been reported. In a recent report, Mertsoylu et al. [12] evaluated $802 \mathrm{NHL}$ patients and found only 2 cases of PTL in Southern Turkey. In an other study from South India, Padhi et al. [13] reported 2 PTL pa- tients among $308 \mathrm{NHL}$ cases. Differently we treated more PTL patients in our department.

PTL is a rare and aggressive form of extranodal NHL which accounts for $1-2 \%$ of $\mathrm{NHL}$ cases in men aged $>60$ years $[1,3]$. Although outcomes for patients with PTL have historically been poor with no plateau in PFS and OS curves, significant improvements have been achieved with the addition of radiotherapy (RT) to fullcourse anthracycline-based chemotherapy, R and CNS-directed prophylaxis [14]. Relapses frequently occur in the contralateral testis and CNS [15]. Both these sites are considered to be immunoprivileged sites, where lymphoma cells may escape the host T-cell antitumour response and chemotherapy may have reduced efficacy because of the mechanical blood testis barrier and blood brain barrier [8,9]. In addition, low levels of p53 expression but high levels of phosphorylated STAT3 (pSTAT3), overexpression of pCXCR4, and upregulation of the nuclear factor kB (NF-kB) pathway were detected in PTL cases by Menter et al. [16] and they reported that expression of both CXCR4 and pCXCR4 was predictive of inferior PFS. Preclinical studies have reported that directed metastasis is mediated by CXCR 4 activation and migration toward CXCR12-expressing target organs [14, 17]. Therefore, CXCR4 overexpression may predispode extranodal relapse in PTL. Due to the above-mentioned mechanisms orchiectomy alone is not sufficient even in the early stages. It has been observed that more than $60 \%$ of patients treated with simple orchiectomy relapsed mostly in the CNS in the first five years $[4,18]$.

In the current study, histopathologically all the patients were DLBCL, which was consistent with other reports [19-21]. The most common regimen reported in retrospective studies is R-cyclophosphamide, doxorubicin, vincristine and prednisolone 3 weekly (R-CHOP-21) and there are no data to suggest that any alternative regimen offers a better outcome [3, 7, 22]. In our study, all the PTL patients received $\mathrm{R}-\mathrm{CHOP}$. $\mathrm{R}$ is a monoclonal antibody against the CD20 antigen expressed on the majority of B-cell lymphomas which has improved the outcome of both high- and low-grade B-cell lymphomas in general. It has also been incorporated into treatment strategies for PTL over the last decade. However, a retrospective analysis of 769 patients with testicular lymphoma from the Surveillance, Epidemiology and End Result database in the USA did not show any improvements in disease-specific survival after 2000, when $\mathrm{R}$ came into common usage [4]. In a randomized trial of 399 patients with DLBCL by Feugier et al. [23] the effect of $\mathrm{CHOP}$ plus $\mathrm{R}$ (R-CHOP) was compared with that of $\mathrm{CHOP}$ and it was shown that the addition of $\mathrm{R}$ did not reduce the risk of dissemination to the CNS at relapse. According to the results of these retrospective studies, the International Extranodal Lymphoma Study Group (IELSG) reported a prospective phase II trial [24], in which 53 patients received $\mathrm{CHOP}-21$ plus $\mathrm{R}$ followed by prophylactic irradiation of the contralateral testis and intrathecal chemotherapy prophylaxis of 4 doses 
of methotrexate. The patients with stage II disease also received involved-field RT. After a median followup of 65 months, the 5 -year PFS and OS rates were $74 \%$ and $85 \%$, respectively. Only 3 cases demonstrated CNS relapse and no cases demonstrated relapse in the contralateral testis.

In the current series, relapse occured in only 1 patient in CNS after 6 months who had not received CNS prophylaxis and he died from his disease 6 months after this relapse. The second patient died because of myoardial infarction 14 months after the diagnosis.

Similar to the IELSG study no contralateral testis relapse was observed in the current study group. Neither was any CNS relapse observed in those who received intratechal methotrexate but the current study population was small and the follow-up period was not long enough for some patients.

As CNS prophylaxis after anthracycline-based combined chemotherapy and RT, Aviles et al. [25] treated 34 PTL patients with high-dose methotrexate $\left(6 \mathrm{~g} / \mathrm{m}^{2}\right)$. More recently Lokesh et al. [26] reported their PTL series but none of them received CNS prophylaxis nor contrlateral testis irradiation so the survival is poor. In an other new report Ichikawa et al. [27] confirmed that combined modality treatment suggests a better PSF similar to our findings.

On the other hand, new potential therapeutic approaches are necessary because of the poor prognosis for relapsed patients especially in CNS. IfCXCR4 is found to have a pathophysiologic role in mediating extranodal relapse, development of the CXCR4 inhibitor may prove attractive as a therapeutic adjuvant to chemotherapy. In addition, overactivation of the NF-kB and STAT3 signalling pathways may be exploited as a target, but rigorous testing is required in prospective clinical trials in PTL patients.

In conclusion, PTL is a rare condition and prospective randomised controlled trials have been difficult to establish. However, collective international experience based on a number of retrospective analyses has led to the evolution of therapeutic protocols that offer a significantly improved prognosis for PTL. The best outcome is achieved from combined treatment with orchiectomy, R-CHOP and CNS prophylaxis with intrathecal chemotherapy and irradiation of the contralateral testis. Despite notable improvement in the PFS and OS, CNS relapse remains a devastating condition. Ongoing IELSG30 phase II trials with modified CNS prophylaxis (intrathecal injections of cytarabine) could yield essential information. In the future, the development of biological therapeutic agents should be considered for this rare but aggressive disease.

\section{ACKNOWLEDGEMENTS}

We thank to Dr. Berkhan Topaktas for his advice on the statistical analyses.

\section{CONFLICT OF INTEREST}

The authors report no conflicts of interest.

\section{REFERENCES}

1. Moller MB, d'Amore F, Christensen BE. Testicular lymphoma: a population-based study of incidence, clinicopathological correlations and prognosis. The Danish Lymphoma Study Group, LYFO. Eur J Cancer 1994; 30A: 1760-4.

2. Wang C, Jiang P, Li J. Primary lymphomas of the prostate: two case reports and a review of the literature. Contemp Oncol (Pozn) 2012; 16: 456-9.

3. Vitolo U, Ferreri AJ, Zucca E. Primary testicular lymphoma. Crit Rev Oncol Hematol 2008; 65: 183-9.

4. Gundrum JD, Mathiason MA, Moore DB, et al. Primary testicular diffuse large B-cell lymphoma: a populationbased study on the incidence, natural history, and survival comparison with primary nodal counterpart before and after the introduction of rituximab. J Clin Oncol 2009; 27: 5227-32.

5. Crellin AM, Hudson BV, Bennett MH, et al. Non-Hodgkin's lymphoma of the testis. Radiother Oncol 1993; 27:99-106.

6. Zouhair A, Herrmann E, Ugurluer G, et al. Primary testicular lymphoma. Swiss Med Wkly 2010; 140: w13076.

7. Seymour JF, Solomon B, Wolf MM, et al. Primary large-cell non-Hodgkin's lymphoma of the testis: a retrospective analysis of patterns of failure and prognostic factors. Clin Lymphoma 2001; 2: 109e115.

8. Riemersma SA, Jordanova ES, Schop RF, et al. Extensive genetic alterations of the HLA region, including homozygous deletions of HLA class II genes in B-cell lymphomas arising in immune-privileged sites. Blood 2000; 96: 3569e-77.

9. Booman M, Douwes J, Glas AM, et al. Mechanisms and effects of loss of human leukocyte antigen class II expression in immune-privileged site-associated B-cell lymphoma. Clin Cancer Res 2006; 12: 2698-705.

10. Bart J, Groen HJ, van der GraafWT, et al. An oncological view on the blood-testis barrier. Lancet Oncol 2002; 3: 357-63.

11. Buskirk SJ, Evans RG, Banks PM, et al. Primary lymphoma of the testis. Int J Radiat Oncol Biol Phys 1982; 8: 1699-703.

12. Mertsoylu H, Muallaoglu S, Besen AA, et al. Primary extranodal non-Hodgkin's lymphoma: clinicopathological features, survival and treatment outcome in two cancer centers of Southern Turkey. Asian Pac J Cancer Prev 2014; 15: 7207-11.

13. Padhi S, Paul TR, Challa S, et al. Primary extra nodal non Hodgkin lymphoma: a 5 year retrospective analysis. Asian Pac J Cancer Prev 2012; 13: 4889-95.

14. Cheah CY, Wirth A, Seymour JF. Primary testicular lymphoma. Blood 2014; 123: 486-93.

15. Fonseca R, Habermann TM, Colgan JP, et al. Testicular lymphoma is associated with a high incidence of extranodal recurrence. Cancer 2000; 88: 154-61.

16. Menter T, Ernst M, Drachneris J, et al. Phenotype profiling of primary testicular diffuse large B-cell lymphomas. Hematol Oncol 2014; 32: 72-81.

17. Domanska UM, Kruizinga RC, Nagengast WB, et al. A review on CXCR4/CXCL12 axis in oncology: no place to hide. Eur J Cancer 2013; 49: 219-30.

18. Bouziani A, M'sakni I, Bougrine F, et al. Non-Hodgkin's malignant lymphoma: a rare cause of testicular tumour. Prog Urol 2004; 14: 1221-3 (in French).

19. Lagrange JL, Ramaioli A, Theodore $\mathrm{CH}$, et al. NonHodgkin's lymphoma of the testis: a retrospective study of 84 patients treated in the French anticancer centres. Ann Oncol 2001; 12: 1313-9.

20. Vural F, Cagirgan S, Saydam G, et al. Primary testicular lymphoma. J Natl Med Assoc 2007; 99: 1277-82.

21. Li D, Xie P, Mi C. Primary testicular diffuse large B-cell lymphoma shows an activated B-cell-like phenotype. Pathol Res Pract 2010; 206: 611-5. 
22. Mazloom A, Fowler N, Medeiros LJ, et al. Outcome of patients with diffuse large B-cell lymphoma of the testis by era of treatment: the M. D. Anderson Cancer Center experience. Leuk Lymphoma 2010; 51: 1217-24.

23. Feugier $\mathrm{P}$, Virion JM, Tilly H, et al. Incidence and risk factors for central nervous system occurrence in elderly patients with diffuse large-B-cell lymphoma: influence of rituximab. Ann Oncol 2004; 15: 129-33.

24. Vitolo U, Chiappella A, Ferreri AJM, et al. First-line treatment for primary testicular diffuse large B-cell lymphoma with rituximab-CHOP, CNS prophylaxis, and contralateral testis irradiation: final results of an international phase II trial. J Clin Oncol 2011; 29: 2766-72.
25. Aviles A, Neri N, Huerta-Guzman J, et al. Testicular lymphoma: organ-specific treatment did not improve outcome. Oncology 2004; 67: 211-4.

26. Lokesh KN, Sathyanarayanan V, Kuntegowdanahalli CL, et al. Primary diffuse large B-cell lymphoma of testis: a single centre experience and review of literature. Urol Ann 2014; 6: 231-4.

27. Ichikawa K, Noguchi M, Koike M, et al. Rituximab plus a CHOP-like regimen, central nervous system prophylaxis, and contralateral testicular irradiation for localized primary testicular diffuse large B-cell lymphoma lead to prolonged progression-free survival. Int J Hematol 2014; 100: $370-8$. 